\title{
Exact Dynamical Exchange-Correlation Kernel of a Weakly Inhomogeneous Electron Gas
}

\author{
V. U. Nazarov, ${ }^{1,2}$ G. Vignale, ${ }^{3}$ and Y.-C. Chang ${ }^{1}$ \\ ${ }^{1}$ Research Center for Applied Sciences, Academia Sinica, Taipei 115, Taiwan \\ ${ }^{2}$ Department of Physical Chemistry, Far-Eastern National Technical University, 10 Pushkinskaya Street, Vladivostok 690950, Russia \\ ${ }^{3}$ Department of Physics and Astronomy, University of Missouri, Columbia, Missouri 65211, USA
}

(Received 26 November 2008; published 18 March 2009)

\begin{abstract}
The dynamical exchange-correlation kernel $f_{\mathrm{xc}}$ of a nonuniform electron gas is an essential input for the time-dependent density-functional theory of electronic systems. The long-wavelength behavior of this kernel is known to be of the form $f_{\mathrm{xc}}=\alpha / q^{2}$ where $q$ is the wave vector and $\alpha$ is a frequency-dependent coefficient. We show that in the limit of weak nonuniformity the coefficient $\alpha$ has a simple and exact expression in terms of the ground-state density and the frequency-dependent kernel of a uniform electron gas at the average density. We present an approximate evaluation of this expression for $\mathrm{Si}$ and discuss its implications for the theory of excitonic effects.
\end{abstract}

Since its introduction in works of Runge, Gross, and Kohn $[1,2]$, the time-dependent density-functional theory (TDDFT) has evolved into a powerful tool of investigation of systems ranging from isolated atoms to bulk solids. In the important linear-response regime, the key quantity of TDDFT is the dynamical exchange-correlation (xc) kernel $f_{\mathrm{xc}}$ defined as the functional derivative

$$
f_{\mathrm{xc}}\left[n_{0}(\mathbf{r})\right]\left(\mathbf{r}, \mathbf{r}^{\prime}, \omega\right)=\left.\frac{\delta V_{\mathrm{xc}}[n](\mathbf{r}, \omega)}{\delta n\left(\mathbf{r}^{\prime}, \omega\right)}\right|_{n=n_{0}(\mathbf{r})}
$$

of the dynamical xc potential $V_{\mathrm{xc}}$ with respect to the dynamical electron density $n$, taken at the ground-state value $n_{0}$ of the latter. With this definition, the densityresponse function $\chi$ can be represented in operator notation as [2]

$$
\chi\left(\mathbf{r}, \mathbf{r}^{\prime}, \omega\right)=\left\{\left[1-\chi_{\mathrm{KS}}\left(C+f_{\mathrm{xc}}\right)\right]^{-1} \chi_{\mathrm{KS}}\right\}\left(\mathbf{r}, \mathbf{r}^{\prime}, \omega\right),
$$

where $\chi_{\mathrm{KS}}$ is the Kohn-Sham (KS) density-response function of independent electrons, $C=e^{2} /\left|\mathbf{r}-\mathbf{r}^{\prime}\right|$ is the Coulomb interaction, and $e$ is the absolute value of the electron charge. While the density-response function of noninteracting electrons $\chi_{\mathrm{KS}}$ can be straightforwardly calculated in many cases of interest [e.g., for homogeneous electron gases (EG) in three and two dimensions it is given by the analytical Lindhard's [3] and Stern's [4] formulas, respectively], the construction of $f_{\mathrm{xc}}$, whose role is to account for dynamical many-body correlations, is a very challenging problem.

As an instructive specific case, let us consider the excitonic effect [5] in a semiconductor, which would manifest itself as an enhancement of the imaginary part of $\chi$ for frequencies close to the fundamental absorption edge. We neglect for a moment local-field effects [6,7] and write down the diagonal elements of the density response in momentum space as of Eq. (1)

$$
\chi(\mathbf{q}, \mathbf{q}, \omega)=\frac{\chi_{\mathrm{KS}}(\mathbf{q}, \mathbf{q}, \omega)}{1-\chi_{\mathrm{KS}}(\mathbf{q}, \mathbf{q}, \omega)\left[\frac{4 \pi e^{2}}{q^{2}}+f_{\mathrm{xc}}(\mathbf{q}, \mathbf{q}, \omega)\right]},
$$

where $4 \pi e^{2} / q^{2}$ is the Fourier transform of the Coulomb interaction. On the one hand, the excitonic enhancement of $\chi$ is a many-body effect and, therefore, it needs a nonzero $f_{\mathrm{xc}}$ to be accounted for within TDDFT. On the other hand, because of the divergent Coulomb part $4 \pi e^{2} / q^{2}$ in Eq. (2), any $f_{\mathrm{xc}}(\mathbf{q}, \mathbf{q}, \omega)$ that remained finite at $q=0$ would give no contribution in the long-wave limit $q \rightarrow 0$. This simple observation shows that in order to include the exciton, $f_{\mathrm{xc}}(\mathbf{q}, \mathbf{q}, \omega)$ must be divergent in the long-wave limit at least as strongly as the Coulomb term; i.e., it must have the form

$$
\lim _{\mathbf{q} \rightarrow 0} f_{\mathrm{xc}}(\mathbf{q}, \mathbf{q}, \omega)=\frac{e^{2} \alpha(\omega)}{q^{2}}
$$

(we introduce the $e^{2}$ so that $\alpha$ is dimensionless). And indeed, when the $q^{-2}$ divergence has been introduced empirically in works dealing with the optical absorption spectrum of semiconductors [8-10], it has yielded a good TDDFT description of the excitonic effect.

From a theoretical standpoint the existence of the singularity in $f_{\mathrm{xc}}(\mathbf{q}, \mathbf{q}, \omega)$ has long been known for periodic insulators both at zero frequency [11] and at finite frequency $[12,13]$. In metallic systems the singularity is believed to be absent at zero frequency, but still present at finite frequency [15]. Unfortunately, first-principle calculations of $\alpha(\omega)$ have not been forthcoming. Kim and Görling [12] have obtained a formal expression for $\alpha(\omega)$ for periodic insulators in the exact exchange approximation to the leading order in the Coulomb interaction-but the evaluation of this expression remains a formidable task. In this Letter we focus on weakly inhomogeneous electronic systems, and develop a theory that is exact to second order in the amplitude of the density modulation. Thus, at variance with the systems considered in Ref. [12], our 
system is a metal and many-body effects are included beyond the exchange-only approximation to all orders in the Coulomb interaction. In particular, we will derive a simple and exact expression for $\alpha(\omega)$ in terms of the ground-state density and the dynamical xc kernel of a homogeneous EG evaluated at the average density of the inhomogeneous system.

Our approach is based on the general method recently derived from the time-dependent current-densityfunctional theory (TDCDFT) for constructing $f_{\mathrm{xc}}$ from the current-current response function [16]. This method is based on the exact relation that connects the scalar density-response function $\chi$ (density response to a scalar potential) to the tensor current-density-response function $\hat{\chi}$ (current-density response to a vector potential):

$$
\chi\left(\mathbf{q}, \mathbf{q}^{\prime}, \omega\right)=\frac{c}{e \omega^{2}} \mathbf{q} \cdot \hat{\chi}\left(\mathbf{q}, \mathbf{q}^{\prime}, \omega\right) \cdot \mathbf{q}^{\prime} .
$$

Both response functions are expressed in terms of the corresponding Kohn-Sham response functions and xc kernels in the following manner:

$$
\chi^{-1}\left(\mathbf{q}, \mathbf{q}^{\prime}, \omega\right)=\chi_{\mathrm{KS}}^{-1}\left(\mathbf{q}, \mathbf{q}^{\prime}, \omega\right)-f_{\mathrm{xc}}\left(\mathbf{q}, \mathbf{q}^{\prime}, \omega\right)-\frac{4 \pi e^{2}}{q^{2}} \delta_{\mathbf{q} \mathbf{q}^{\prime}}
$$

and

$\hat{\chi}^{-1}\left(\mathbf{q}, \mathbf{q}^{\prime}, \omega\right)=\hat{\chi}_{\mathrm{KS}}^{-1}\left(\mathbf{q}, \mathbf{q}^{\prime}, \omega\right)-\hat{f}_{\mathrm{xc}}\left(\mathbf{q}, \mathbf{q}^{\prime}, \omega\right)-\frac{4 \pi e c}{\omega^{2}} \hat{L}_{\mathbf{q}} \delta_{\mathbf{q q}}$,

where $\hat{L}_{\mathbf{q}, i j} \equiv q_{i} q_{j} / q^{2}, i$, and $j$ are Cartesian indices.

Equations (4)-(6) establish a connection between $f_{\mathrm{xc}}$ and its tensor counterpart $\hat{f}_{\mathrm{xc}}$. The usefulness of this connection stems from the fact that the tensor quantities $\hat{\chi}_{\mathrm{KS}}$ and $\hat{f}_{\mathrm{xc}}$ satisfy a broader set of exact sum rules than the corresponding scalar quantities. These sum rules were derived in Ref. [17]. Specializing to the case of periodic systems, the two most important sum rules for our purposes are

$$
\begin{aligned}
\hat{\chi}_{\mathrm{KS}, i j}(\mathbf{G}, \mathbf{0}, \omega)= & \frac{e}{m c} n_{0}(\mathbf{G}) \delta_{i j}-\frac{1}{m \omega^{2}} \\
& \times \sum_{\mathbf{G}^{\prime}, k} \hat{\chi}_{\mathrm{KS}, i k}\left(\mathbf{G}, \mathbf{G}^{\prime}, \omega\right) G_{k}^{\prime} G_{j}^{\prime} V_{\mathrm{KS}}\left(\mathbf{G}^{\prime}\right),
\end{aligned}
$$

and

$$
\sum_{\mathbf{G}^{\prime}} \hat{f}_{\mathrm{xc}, i j}\left(\mathbf{G}, \mathbf{G}^{\prime}, \omega\right) n_{0}\left(\mathbf{G}^{\prime}\right)=\frac{c}{e \omega^{2}} G_{i} G_{j} V_{\mathrm{xc}}(\mathbf{G}),
$$

where $\mathbf{G}$ are reciprocal lattice vectors. These sum rules connect three different types of components of, say, $\hat{\chi}_{\mathrm{KS}}\left(\mathbf{G}, \mathbf{G}^{\prime}, \omega\right)$ : the $(\mathbf{0}, \mathbf{0})$ (head), the $(\mathbf{0}, \mathbf{G} \neq \mathbf{0})$ and $(\mathbf{G} \neq$ $\mathbf{0}, \mathbf{0})$ (wing), and the $\left(\mathbf{G} \neq \mathbf{0}, \mathbf{G}^{\prime} \neq \mathbf{0}\right)$ (body) components.

Let us further restrict our attention to the case of a weakly inhomogeneous system: $\left|n_{0}(\mathbf{G})\right| \ll n_{0}(\mathbf{0}) \equiv \bar{n}_{0}$, and $\left|V_{\mathrm{KS}}(\mathbf{G})\right| \ll \hbar^{2} G^{2} / 2 m$ for $\mathbf{G} \neq \mathbf{0}$. Then Eq. (7) leads to

$$
\begin{aligned}
\hat{\chi}_{\mathrm{KS}, i j}\left(\mathbf{G} \neq \mathbf{0}, \mathbf{G}^{\prime} \neq \mathbf{0}, \omega\right)= & {\left[\frac{e \omega^{2}}{c G^{2}} L_{\mathbf{G}, i j} \chi_{\mathrm{KS}}^{h L}(G, \omega)\right.} \\
& \left.+T_{\mathbf{G}, i j} \chi_{\mathrm{KS}}^{h T}(G, \omega)\right] \delta_{\mathbf{G G}^{\prime},}
\end{aligned}
$$

$$
\begin{aligned}
\hat{\chi}_{\mathrm{KS}, i j}(\mathbf{G} \neq \mathbf{0}, \mathbf{0}, \omega)= & \hat{\chi}_{\mathrm{KS}, j i}(\mathbf{0},-\mathbf{G}, \omega) \\
= & \frac{e n_{0}(\mathbf{G})}{m c}\left[\delta_{i j}-\frac{\chi_{\mathrm{KS}}^{h L}(G, \omega)}{\chi_{\mathrm{KS}}^{h L}(G, 0)} L_{\mathbf{G}, i j}\right], \\
\hat{\chi}_{\mathrm{KS}, i j}(\mathbf{0}, \mathbf{0}, \omega)= & \frac{e \bar{n}_{0}}{m c} \delta_{i j}+\frac{e}{m^{2} \omega^{2} c} \sum_{\mathbf{G} \neq \mathbf{0}} \frac{\left|n_{0}(\mathbf{G})\right|^{2}}{\chi_{\mathrm{KS}}^{h L}(G, 0)^{2}} \\
& \times\left[\chi_{\mathrm{KS}}^{h L}(G, \omega)-\chi_{\mathrm{KS}}^{h L}(G, 0)\right] G_{i} G_{j},
\end{aligned}
$$

to the zeroth, first, and second order in $n_{0}(\mathbf{G} \neq 0)$, respectively. Here $\chi_{\mathrm{KS}}^{h L}$ and $\chi_{\mathrm{KS}}^{h T}$ are, respectively, the longitudinal and transverse KS density-response functions of the homogeneous EG of density $\bar{n}_{0}$, and $T_{\mathbf{G}, i j}=$ $\delta_{i j}-L_{\mathbf{G}, i j}$. Equation (10) is obtained by the substitution for $\sum_{k} \hat{\chi}_{\mathrm{KS}, i k}\left(\mathbf{G} \neq \mathbf{0}, \mathbf{G}^{\prime} \neq \mathbf{0}, \omega\right) G_{k}$ in the right-hand side of Eq. (7) of its homogeneous value of $\left(e \omega^{2} G_{i} / c G^{2}\right) \chi_{\mathrm{KS}}^{h L}(G, \omega) \delta_{\mathbf{G G}^{\prime}}$. Equation (11) is obtained by the substitution of Eq. (10) into the right-hand side of Eq. (7) after setting $\mathbf{G}=0$. Equation (9) just replaces $\hat{\chi}_{\mathrm{KS}}\left(\mathbf{G} \neq \mathbf{0}, \mathbf{G}^{\prime} \neq \mathbf{0}, \omega\right)$ by its homogeneous value. The relation $V_{\mathrm{KS}}(\mathbf{G} \neq 0)=n_{0}(\mathbf{G}) / \chi_{K S}^{h L}(G, 0)$ valid to the first order in $n_{0}(\mathbf{G} \neq 0)$ has been used. Similarly, for $\hat{f}_{\mathrm{xc}}$ we have from Eq. (8)

$$
\begin{aligned}
\hat{f}_{\mathrm{xc}, i j}\left(\mathbf{G} \neq 0, \mathbf{G}^{\prime} \neq 0, \omega\right)= & \frac{c}{e \omega^{2}} G^{2}\left[f_{\mathrm{xc}}^{h L}(G, \omega) L_{\mathbf{G}, i j}\right. \\
& \left.+f_{\mathrm{xc}}^{h T}(G, \omega) T_{\mathbf{G}, i j}\right] \delta_{\mathbf{G G}}, \\
\hat{f}_{\mathrm{xc}, i j}(\mathbf{G} \neq \mathbf{0}, \mathbf{0}, \omega)= & \hat{f}_{\mathrm{xc}, j i}(\mathbf{0},-\mathbf{G}, \omega) \\
= & -\frac{c G^{2}}{e \omega^{2} \bar{n}_{0}} n_{0}(\mathbf{G})\left\{\left[f_{\mathrm{xc}}^{h L}(G, \omega)\right.\right. \\
& \left.\left.-f_{\mathrm{xc}}^{h L}(G, 0)\right] L_{\mathbf{G}, i j}+f_{\mathrm{xc}}^{h T}(G, \omega) T_{\mathbf{G}, i j}\right\}, \\
\hat{f}_{\mathrm{xc}, i j}(\mathbf{0}, \mathbf{0}, \omega)= & \frac{c}{e \omega^{2} \bar{n}_{0}^{2}} \sum_{\mathbf{G} \neq \mathbf{0}} G^{2}\left|n_{0}(\mathbf{G})\right|^{2}\left\{\left[f_{\mathrm{xc}}^{h L}(G, \omega)\right.\right. \\
& \left.\left.-f_{\mathrm{xc}}^{h L}(G, 0)\right] L_{\mathbf{G}, i j}+f_{\mathrm{xc}}^{h T}(G, \omega) T_{\mathbf{G}, i j\}}\right\},
\end{aligned}
$$

where $f_{\mathrm{xc}}^{h L}$ and $f_{\mathrm{xc}}^{h T}$ are the longitudinal and transverse, respectively, xc kernels of the homogeneous EG of density $\bar{n}_{0}$.

The following steps, which involve repeated inversions of infinite matrices, rely on the mathematical fact that to find the head, wing, and body elements of the inverse matrix to the second, first, and zeroth order in the inhomogeneity, respectively, it is sufficient to know the corresponding elements of the original matrix to the same 
orders, and then the inversion can be performed in a closed form (cf., Ref. [18]). The complete procedure is schematically illustrated in Fig. 1. Starting from Eqs. (9)-(14) for $\hat{\chi}_{\mathrm{KS}}$ and $\hat{f}_{\mathrm{xc}}$ we (i) invert Eqs. (9)-(11) to get $\hat{\chi}_{\mathrm{KS}}^{-1}$; (ii) combine $\hat{\chi}_{\mathrm{KS}}^{-1}$ and $\hat{f}_{\mathrm{xc}}$ to get $\hat{\chi}^{-1}$ by virtue of Eq. (6); (iii) invert $\hat{\chi}^{-1}$ to get $\hat{\chi}$; (iv) use Eq. (4) and its KS analogue to find the scalar response function $\chi$ from $\hat{\chi}$ and $\chi_{\mathrm{KS}}$ from $\hat{\chi}_{\mathrm{KS}}$; (v) invert $\chi$ and $\chi_{\mathrm{KS}}$ to get $\chi^{-1}$ and $\chi_{\mathrm{KS}}^{-1}$; and, (vi) apply Eq. (5) to find $f_{\mathrm{xc}}$. We note that in our analysis we are remaining within the domain of the equivalence between TDCDFT and TDDFT, keeping $\mathbf{q}$ small but finite [19]. The final result of this procedure is

$$
\begin{aligned}
f_{\mathrm{xc}}\left(\mathbf{G} \neq \mathbf{0}, \mathbf{G}^{\prime} \neq \mathbf{0}, \omega\right)=f_{\mathrm{xc}}^{h L}(G, \omega) \delta_{\mathbf{G G}^{\prime}}, \\
\begin{aligned}
\lim _{q \rightarrow 0} f_{\mathrm{xc}}(\mathbf{G} \neq \mathbf{0}, \mathbf{q}, \omega)= & f_{\mathrm{xc}}(-\mathbf{q},-\mathbf{G}, \omega) \\
= & -\frac{(\mathbf{G} \cdot \hat{\mathbf{q}})}{\bar{n}_{0} q}\left[f_{\mathrm{xc}}^{h L}(G, \omega)\right. \\
& \left.-f_{\mathrm{xc}}^{h L}(G, 0)\right] n_{0}(\mathbf{G}), \\
\lim _{q \rightarrow 0} f_{\mathrm{xc}}(\mathbf{q}, \mathbf{q}, \omega)= & \frac{1}{\bar{n}_{0}^{2} q^{2}} \sum_{\mathbf{G} \neq \mathbf{0}}(\mathbf{G} \cdot \hat{\mathbf{q}})^{2}\left[f_{\mathrm{xc}}^{h L}(G, \omega)\right. \\
& \left.-f_{\mathrm{xc}}^{h L}(G, 0)\right]\left|n_{0}(\mathbf{G})\right|^{2},
\end{aligned}
\end{aligned}
$$

where $\hat{\mathbf{q}}$ is the unit vector parallel to $\mathbf{q}$. It should be noted at this point that the above expression for the scalar kernel $f_{\mathrm{xc}}(\mathbf{q}, \mathbf{q}, \omega)$ differs from what one would get by simply taking the longitudinal component of $\hat{f}_{\mathrm{xc}, i j}(\mathbf{q}, \mathbf{q}, \omega)$, i.e., $f_{\mathrm{xc}}(\mathbf{q}, \mathbf{q}, \omega) \neq \frac{e \omega^{2}}{c q^{2}} \sum_{i, j} \hat{q}_{i} \hat{f}_{\mathrm{xc}, i j}(\mathbf{q}, \mathbf{q}, \omega) \hat{q}_{j}$. The implication is that the scalar xc potential $\left(V_{\mathrm{xc}}\right)$ of time-dependent DFT is not equivalent to the longitudinal component of the vector potential $\left(\mathbf{A}_{\mathrm{xc}}\right)$ of time-dependent CDFT: rather, it should be constructed through the careful inversion procedure described above. A recent interesting attempt to construct $V_{\text {xc }}$ from $\mathbf{A}_{\text {xc }}$ [20] should be reexamined in the light of this result.

From the result of the step (iv) for $\chi$ we obtain a formula for the macroscopic dielectric function of a crystal

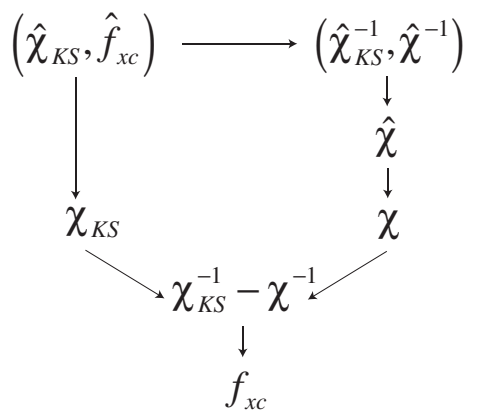

FIG. 1. Scheme of the procedure for calculating the xc kernel $f_{\mathrm{xc}}$ starting from the expressions (9)-(14) for $\hat{\chi}_{\mathrm{KS}}$ and $\hat{f}_{\mathrm{xc}}$.

$$
\begin{aligned}
\lim _{q \rightarrow 0} \epsilon_{M}(\mathbf{q}, \omega)= & 1-\frac{4 \pi e^{2} \bar{n}_{0}}{m \omega^{2}}-\frac{e^{2}}{m^{2} \omega^{4}} \sum_{\mathbf{G} \neq 0}\left|V_{0}(\mathbf{G})\right|^{2} G^{2}(\hat{\mathbf{q}} \cdot \mathbf{G})^{2} \\
& \times\left[\frac{1}{\epsilon^{h L}(G, \omega)}-\frac{1}{\epsilon^{h L}(G, 0)}\right]
\end{aligned}
$$

where $V_{0}$ is the bare crystalline potential and $\epsilon^{h L}(q, \omega)$ is the longitudinal dielectric function of the homogeneous electron liquid. Equation (18) is in agreement with the Hopfield's formula for optical conductivity [21], and reduces to the corresponding results of Refs. $[7,18]$ in the random phase approximation $\left[f_{\mathrm{xc}, L}^{h}(G, \omega)=0\right]$.

Equations (15)-(17) are the main result of this Letter. In the inhomogeneous case they replace the grossly inaccurate LDA formula [2,22-24] which does not contain the singularity in $q$. Identifying the $(\mathbf{0}, \mathbf{0})$ element of the microscopic matrix of the xc kernel in Eq. (17) as the averaged $f_{\mathrm{xc}}$, we see that $f_{\mathrm{xc}}$ diverges for $q \rightarrow 0$ as described by Eq. (3), wherein $\alpha(\omega)$ is given by [25]

$$
\alpha(\omega)=\sum_{\mathbf{G} \neq 0} \frac{(\mathbf{G} \cdot \hat{\mathbf{q}})^{2}}{e^{2} \bar{n}_{0}^{2}}\left[f_{\mathrm{xc}}^{h L}(G, \omega)-f_{\mathrm{xc}}^{h L}(G, 0)\right]\left|n_{0}(\mathbf{G})\right|^{2} .
$$

We notice that $\alpha(\omega)=0$ in the uniform limit and $\alpha(0)=0$ up to second order in $n_{0}(\mathbf{G} \neq \mathbf{0})$. This shows that the $q^{-2}$ singularity is absent at zero frequency in systems that are perturbatively connected to the homogeneous electron liquid, i.e., metals [26].

In order to calculate $\alpha(\omega)$ from Eq. (19) we need the Fourier amplitudes of the ground-state electron density and the wave vector and frequency-dependent $f_{\mathrm{xc}}^{h L}$ of the homogeneous EG, evaluated at reciprocal lattice vectors. The first ingredient is straightforwardly obtained from standard electronic structure calculations. Unfortunately, the same cannot be said of the second ingredient $f_{\mathrm{xc}}^{h L}(q, \omega)$ : A reliable $q$ - and $\omega$-dependent xc kernel of the homogeneous EG which reproduces, at small $q$, what is presently believed to be the qualitatively correct form of the frequency dependence is not known to us. In spite of these difficulties, it must be emphasized that the calculation of $f_{\mathrm{xc}}^{h L}(q, \omega)$ is still a much simpler problem than the calculation of the dynamical xc kernel of the nonuniform system. Thus, our Eq. (19) does not simply express an unknown quantity in terms of another unknown quantity, but actually opens the way to systematic calculations of $\alpha$ based on the many-body theory of the homogeneous EG. Further, Eqs. (15)-(17) for $f_{\mathrm{xc}}$ offer a promising alternative to the widespread practice of treating the dynamical xc effects in the LDA.

In Fig. 2, we plot $\alpha(\omega)$ from Eq. (19) vs frequency for crystalline silicon. The Fourier coefficients of the electron density were calculated with the code FHI98MD [27], and we approximated $f_{\mathrm{xc}}^{h L}(q, \omega) \simeq f_{\mathrm{xc}}^{h L}(0, \omega)$, taking the latter from Ref. [24]. In the range $0-22 \mathrm{eV}$, the real part of $\alpha(\omega)$ is negative, changing sign for positive above $22 \mathrm{eV}$. It reaches its minimum of $\alpha \approx-0.1$ at $\omega \approx 14 \mathrm{eV}$. In the range $3-5 \mathrm{eV}$ of the main absorption in silicon, $\operatorname{Re} \alpha$ 

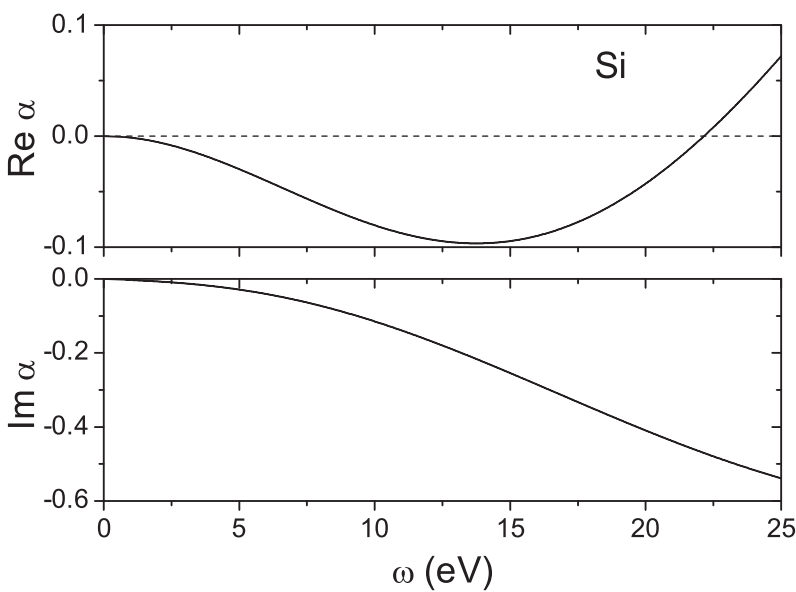

FIG. 2. The frequency dependence of the real (upper panel) and imaginary (lower panel) parts of the $\alpha$ coefficient in Eq. (3) for silicon calculated by Eq. (19).

changes from -0.01 to -0.03 , which is an order of magnitude smaller than the empirical value of $\alpha \approx-0.2$ found as the best fit to the experimental spectrum in Ref. [8]. This large difference may simply indicate that the nearly free electron model, while being adequate for simple metals and even for semiconductors in the high-frequency regime [18], is not sufficiently accurate for semiconductors at frequency lower than or comparable to the band gap. Another probable source of discrepancy is that our approach is a pure TDDFT, whereas the value of $\alpha \approx-0.2$ was obtained in Refs. [8,9] with the use of TDDFT together with the self-energies incorporated in the Green's function via the $G W$ approximation.

In conclusion, we wish to comment on the relation between the present exact results and the approximate nonlocal $f_{\mathrm{xc}}$ that can be derived from the Vignale-Kohn (VK) local density approximation to TDCDFT $[15,17,28]$. The VK approximation is applicable in principle to strongly inhomogenous systems (e.g., insulators), provided the density is slowly varying on the scale of the interparticle distance and $\omega \gg \max \left(q v_{F}, G v_{F}\right)$ where $v_{F}$ is the local Fermi velocity. In contrast, our Eq. (17) is only valid when the inhomogeneity is weak. When the inhomogeneity is both weak and slowly varying and $\omega \gg$ $\max \left(q v_{F}, G v_{F}\right)$, then our formula (17) is indeed equivalent to the VK approximation as it should.

A clear advantage of our formula is that it is based on controlled approximations. We believe that this formula will be useful in the study of the dynamical response of metals at all frequencies, and of insulators at not too low frequencies, provided the nonuniformity of the valence electron density is small.

G. V. acknowledges support from DOE Grant No. DEFG02-05ER46203.
[1] E. Runge and E. K. U. Gross, Phys. Rev. Lett. 52, 997 (1984).

[2] E. K. U. Gross and W. Kohn, Phys. Rev. Lett. 55, 2850 (1985).

[3] J. Lindhard, K. Dan. Vidensk. Selsk. Mat.-Fys. Medd. 28, 1 (1954).

[4] F. Stern, Phys. Rev. Lett. 18, 546 (1967).

[5] N.W. Ashcroft and N.D. Mermin, Solid State Physics (Holt, Rinehart and Winston, New-York, 1976).

[6] S. L. Adler, Phys. Rev. 126, 413 (1962).

[7] N. Wiser, Phys. Rev. 129, 62 (1963).

[8] L. Reining, V. Olevano, A. Rubio, and G. Onida, Phys. Rev. Lett. 88, 066404 (2002).

[9] R. Del Sole, G. Adragna, V. Olevano, and L. Reining, Phys. Rev. B 67, 045207 (2003).

[10] S. Botti, F. Sottile, N. Vast, V. Olevano, L. Reining, H.-C. Weissker, A. Rubio, G. Onida, R. Del Sole, and R. W. Godby, Phys. Rev. B 69, 155112 (2004).

[11] P. Ghosez, X. Gonze, and R. W. Godby, Phys. Rev. B 56, 12811 (1997).

[12] Y.-H. Kim and A. Görling, Phys. Rev. B 66, 035114 (2002).

[13] A closely related point of the singularity in $q$ of the inverse density-response function of crystals had been under discussion in the pre-TDDFT era [6,7,14].

[14] R. M. Pick, M. H. Cohen, and R. M. Martin, Phys. Rev. B 1, 910 (1970).

[15] G. Vignale and W. Kohn, Phys. Rev. Lett. 77, 2037 (1996).

[16] V. U. Nazarov, J. M. Pitarke, Y. Takada, G. Vignale, and Y.-C. Chang, Phys. Rev. B 76, 205103 (2007).

[17] G. Vignale and W. Kohn, in Electronic Density Functional Theory: Recent Progress and New Directions, edited by J. Dobson, M.P. Das, and G. Vignale (Plenum Press, New York, 1998).

[18] K. Sturm, Adv. Phys. 31, 1 (1982).

[19] N. T. Maitra, I. Souza, and K. Burke, Phys. Rev. B 68, 045109 (2003).

[20] N. T. Maitra and M. van Faassen, J. Chem. Phys. 126, 191106 (2007).

[21] J. J. Hopfield, Phys. Rev. 139, A419 (1965).

[22] R. Nifosì, S. Conti, and M. P. Tosi, Phys. Rev. B 58, 12758 (1998).

[23] S. Conti and G. Vignale, Phys. Rev. B 60, 7966 (1999).

[24] Z. Qian and G. Vignale, Phys. Rev. B 65, 235121 (2002).

[25] We have checked that expression (19), evaluated to the first order in the Coulomb interaction, is consistent with the formulas of Ref. [12] if the latter are expanded to second order in the inhomogeneous density.

[26] Even though in our derivation we let $\mathbf{q}$ tend to zero before $\omega$, we have checked that the same result is obtained if the order of the limits is reversed.

[27] M. Bockstedte, A. Kley, J. Neugebauer, and M. Scheffler, Comput. Phys. Commun. 107, 187 (1997); code FHI98MD is available at http://www.fhi-berlin.mpg.de/th/fhi98md/.

[28] G. Vignale, C. A. Ullrich, and S. Conti, Phys. Rev. Lett. 79, 4878 (1997). 